\title{
Response Accuracy and Tracking Errors with Decentralized Control of Commercial V2G Chargers
}

Ziras, Charalampos; Zecchino, Antonio; Marinelli, Mattia

Published in:

Proceedings of 20th Power System Computation Conference

Link to article, DOI:

10.23919/PSCC.2018.8442488

Publication date:

2018

Document Version

Peer reviewed version

Link back to DTU Orbit

Citation (APA):

Ziras, C., Zecchino, A., \& Marinelli, M. (2018). Response Accuracy and Tracking Errors with Decentralized Control of Commercial V2G Chargers. In Proceedings of 20th Power System Computation Conference IEEE. https://doi.org/10.23919/PSCC.2018.8442488

\section{General rights}

Copyright and moral rights for the publications made accessible in the public portal are retained by the authors and/or other copyright owners and it is a condition of accessing publications that users recognise and abide by the legal requirements associated with these rights.

- Users may download and print one copy of any publication from the public portal for the purpose of private study or research.

- You may not further distribute the material or use it for any profit-making activity or commercial gain

- You may freely distribute the URL identifying the publication in the public portal 


\title{
Response Accuracy and Tracking Errors with Decentralized Control of Commercial V2G Chargers
}

\author{
Charalampos Ziras, Antonio Zecchino, Mattia Marinelli \\ Center for Electric Power and Energy - Department of Electrical Engineering \\ DTU - Technical University of Denmark \\ Ris $\varnothing$ Campus, Roskilde, Denmark \\ \{chazi, antozec, matm\}@elektro.dtu.dk
}

\begin{abstract}
There is a growing interest in using the flexibility of electric vehicles (EVs) to provide power system services, such as fast frequency regulation. Decentralized control is advocated due to its reliability and much lower communication requirements. A commonly used linear droop characteristic results in low average efficiencies, whereas controllers with 3 modes (idle, fully charging, fully discharging) result in large reserve errors when the aggregation size is small. To address these issues, we propose a stochastic, decentralized controller with tunable response granularity which minimizes switching actions. The EV fleet operator can optimize the chargers' performance according to the fleet size, the service error requirements, the average switching rate and the average efficiency. We use real efficiency characteristics from EVs and chargers providing fast frequency regulation and we show that the proposed controller can significantly reduce reserve errors and increase efficiency for a given fleet size, while at the same time minimizing the switching actions.
\end{abstract}

Index Terms-Decentralized control, electric vehicles, primary frequency control, stochastic control, V2G chargers.

\section{INTRODUCTION}

Electric vehicles (EVs) are recognized as an important source of load flexibility and as a potential provider of power systems services in the context of vehicle to grid (V2G) technologies. A suitable service for EVs is primary frequency control (PFC), due to the chargers' high power capacity and very fast response, as well as the relatively low energy requirements of this service. EVs' technical capabilities in providing different ancillary services including PFC have been experimentally proven both at a microgrid level and on a real distribution network [1], [2]. Even though a single EV's capacity is not particularly large compared to generators, if a large number of EVs is controlled by an aggregator, it is possible to offer significant amounts of reserve capacity. The literature proposes aggregate models and control schemes for both centralized [3], [4] and decentralized [5]-[8] solutions for the optimal management of EV fleets performing frequency control.

A centralized controller was proposed in [4] for offering secondary frequency control, where a discretized regulation logic is utilized, aiming at meeting the desired calculated total power signal by turning certain EVs on or off according to a priority index. More advanced control strategies have also been proposed to track reference signals with EVs, considering uncertainties and charging efficiencies [9]. However, as the control architecture is centralized, real-time communication is required, which may result in high infrastructure costs, as well as in loss of controllability in case of an outage of the communication system. Due to the critical nature of PFC to a power system's stability and stricter requirements than secondary control, very high reliability and very low latencies are required.

Such risks are highly reduced in the case of decentralized EV control, as decentralized PFC offers higher reliability and significantly reduces the communication requirements compared to real-time centralized control. A decentralized stochastic control component is proposed in [3], where the decision to change the charging set-point is taken locally by the EVs, even though with a remote centralized frequency measurement performed by the aggregator, who will dispatch the same correspondent signal to the EVs of the portfolio. In [6] it is shown how demand can respond to frequency deviations in a manner similar to the generators in a purely decentralized way, making it a significant and reliable asset as contribution to PFC. In [5] optimal EV droop curves are designed to improve system stability and in [10] adaptive droops are proposed for EVs offering PFC, to take state of charge (SOC) requirements into account. Finally, [7] proposes a distributed frequency control method, which randomly assigns delays to each EV of the fleet, aiming at avoiding problems to the power system in case of high shares of EVs providing regulation and simultaneous response of all units to the same frequency signals.

However, all the mentioned works do not consider the implications of using droop curves with regards to reserve errors, average charging efficiency, average equipment switching rates and aggregation size when offering PFC in a decentralized way. The commonly used droop-curve characteristic that EVs must follow to provide PFC results in a low average efficiency because of the low loadings of the inverters in most cases. Additionally, as we show in Section IV, a deterministic response always results in reserve errors due to the ISO 15118 , ICE 61851 standard requirement of 1 A granularity when setting the charging rate of the inverter [11]. A stochastic controller (where EVs alternate between idle and full response stochastically and do not respond linearly to the frequency deviation) can significantly increase the efficiency, albeit the resulting errors depend on the aggregation size. 
The main contribution of this paper is the investigation of the trade-off between service accuracy and efficiency under stochastic decentralized control. More specifically, we propose a stochastic controller with a varying number of states, as a trade-off between accuracy and efficiency, which the aggregator can tune depending on the size of the fleet and the service requirements. We show the dependency of the reserve error on the aggregation size and the controller's tuning and we determine the minimum amount of EVs to guarantee a service provision error. Additionally, we calculate the efficiencies achieved with each controller tuning, using real data of $\mathrm{V} 2 \mathrm{G}$ chargers from ENEL, which are currently installed in Denmark and offer fast frequency control grid services [12].

The proposed method allows an EV aggregator to maximize efficiency for a specified number of EVs, by respecting the average reserve error requirements of the provided service. Additionally, we propose a modified version of the control algorithm which decreases the switching rate of the inverters, a modification which can potentially reduce the wear of the components. We show via simulations that our controller significantly increases the service accuracy of the droopbased control, under the 1 A granularity limitation, even for very small aggregation sizes, and that much higher average efficiencies can be achieved for smaller aggregation sizes, when a 3 mode response (idle, full charge, full discharge) results in large reserve errors.

The remainder of the paper is structured as follows. Section II introduces the principles of the frequency-controlled normal operation reserve service and frequency control with EVs. Section III presents an efficiency characteristic from operational V2G chargers. In Section IV we present the proposed discretized, stochastic decentralized controller. In Section V simulation results are presented and discussed. Conclusions are reported in Section VI.

\section{FREQUENCY CONTROL WITH EVS}

Fast frequency control, i.e. PFC, can take different forms depending on the implementation of each Transmission System Operator (TSO). In the Regional Group Nordic (RGN) synchronous area, PFC consists of two separate services, namely frequency-controlled normal operation reserve (FNR), which is activated linearly for all system frequency deviations up to $\pm 100 \mathrm{mHz}$ and frequency-controlled disturbance reserve (FDR), activated only when system frequency drops below $49.9 \mathrm{~Hz}$. We are focusing on FNR, since the revenue potential is higher and this service is currently being provided by commercial V2G chargers in Denmark within a pilot project [12].

In the case of a frequency deviation, the purpose of FNR is to react quickly and try to contain the frequency deviation. The TSOs in RG-N are jointly responsible for procuring 600 MW of FNR reserves, which are divided proportionally to each TSO. FNR is a symmetrical service, which means that the provider must offer the same upwards and downwards reserve capacity. Frequency reserve is provided linearly, with full activation for deviations of $\pm 100 \mathrm{mHz}$. According to the service requirements, response has to be provided linearly and deployed within 150 seconds [13]. These requirements are designed for slower-acting conventional power plants; instead, we consider instant reserve activation reserve in the case of V2G chargers, because this can significantly improve system performance.

For a frequency value $f_{t}$ at time $t$, the normalised requested load $P_{\text {req, } t}$ is calculated as

$$
P_{\text {req }, t}=\left\{\begin{array}{cl}
-1 & \text { if } f_{t}<49.9 \mathrm{~Hz} \\
\left(f_{t}-50\right) / 0.1 & \text { if } 49.9 \mathrm{~Hz} \leq f_{t} \leq 50.1 \mathrm{~Hz} \\
1 & \text { if } f_{t}>50.1 \mathrm{~Hz}
\end{array}\right.
$$

By normalized response we refer to the reserve capacity, $P_{\text {res }}$, of a service provider. As already explained, there are two ways that an aggregation of EVs can modulate its load to provide FNR. In a centralized control scheme the aggregator will calculate the required change in the aggregate load of the EVs and send signals to the individual EVs when it is required. These signals may correspond to deterministic commands, i.e. explicit set-points, or signals containing switching probabilities. In the latter case, the EV will draw a random number and decide to change its set-point or not [5]. However, in these approaches very advanced and reliable real-time communication is required.

In a decentralized control scheme, each EV measures frequency locally and changes its set-point based on a control logic and the individual reserve capacity assigned to it. Other control layers can periodically modify each EV's reserve capacity or target set-point, i.e. the operating set-point when no reserve is offered, on longer time scales based on various parameters. This control structure, which adjusts each EV's reserve capacity and target set-point on a longer time scale (e.g. 15 minutes), while each EV responds based on local measurements, can reduce the communication requirements significantly and retain the robustness of reserve provision. The decentralized nature of reserve provision is thus maintained and EVs respond by only measuring local frequency, whereas the upper control layer can make adjustments on the reserve capacity and target set-point. In this paper we focus on the lower, decentralized control layer assuming that the target set-point is equal to zero and $P_{\text {res }}$ is symmetrical and equal to the maximum capability of each EV. In our future work we will generalize our method by considering arbitrary target setpoints and non-symmetrical assigned reserve capacities. The most standardized, simple and common control method is for a charger to respond linearly to frequency deviations based on a droop curve, as shown in Fig. 1 [14] for a charger with a capacity of $\pm 25 \mathrm{~A}$.

Due to the 1 A response granularity, some reserve errors will occur because the requested response $P_{\text {req, } t}$ is rounded to the nearest corresponding power value. This is an inherent limitation of the response granularity (the implemented droop curve cannot match the ideal one), but these errors can be significantly reduced if a stochastic controller is introduced, as explained in Section IV. At this point we must introduce 


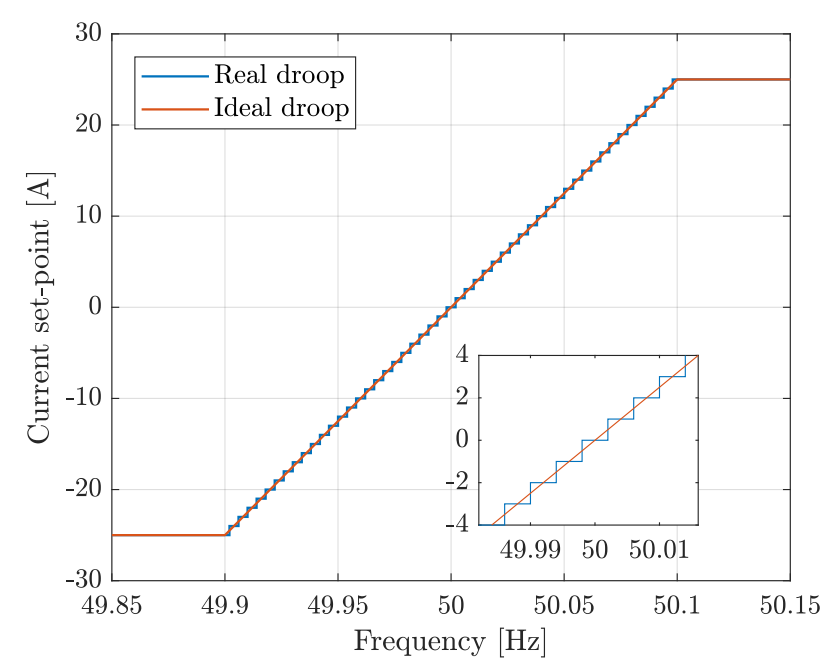

Figure 1: Real and ideal droop curves with 1 A granularity.

a metric to assess the accuracy of the various controllers. Without loss of generality, we consider the case where all EVs (with $i$ being the index of $\mathrm{N} \mathrm{EVs)} \mathrm{offer} \mathrm{the} \mathrm{same} \mathrm{reserve}$ capacity and we denote by $y_{t}^{i}$ the actual, normalized load of EV $i$ at step $t$. A deterministic controller will then result to the same error for all EVs, assuming that the measured $f_{t}$ is the same for the whole aggregation, which is a realistic assumption as long as the aggregated set of chargers is not too geographically dispersed. If each $\mathrm{EV}$ is offering a different reserve capacity, then their contributions must be weighted appropriately. For simplicity we use normalized variables, i.e. on the maximum charger capacity which is equal to $10 \mathrm{~kW}$, and for a period of $t_{\text {tot }}$ the mean average percentage error (MAPE) will be equal to

$$
M A P E[\%]=\frac{\sum_{i=1}^{\mathrm{N}} \sum_{t=1}^{t_{\text {tot }}}\left|y_{t}^{i}-P_{\mathrm{req}, t}^{i}\right|}{\mathrm{N} t_{\mathrm{tot}}} \cdot 100 \%
$$

\section{V2G CHARGER EFFICIENCY CHARACTERISTIC}

An EV performing FNR in a decentralized manner is expected to continuously alternate between charging and discharging modes to follow the frequency deviations and provide reserve power. Apart from the battery degradation that this may cause (and the associated costs), efficiency losses may significantly affect the economic performance of an aggregator performing this service. As we will show next, the way EVs perform FNR has a considerable impact on the efficiency losses during reserve provision. In Fig. 2 a V2G charger efficiency characteristic is presented, which was derived by real data from EVs performing FNR [14].

One can notice that efficiency is considerably lower for small loadings because the inverter is designed to operate more efficiently closer to the maximum loading values. In Fig. 3 a histogram of 10 days of frequency values for 2016 is presented, where it is evident that most frequency samples lie within a narrow band around $50 \mathrm{~Hz}$. The frequency data corresponds to real frequency measurements of RG-N area from the Norwegian TSO [15]. Approximately $85 \%$ of the

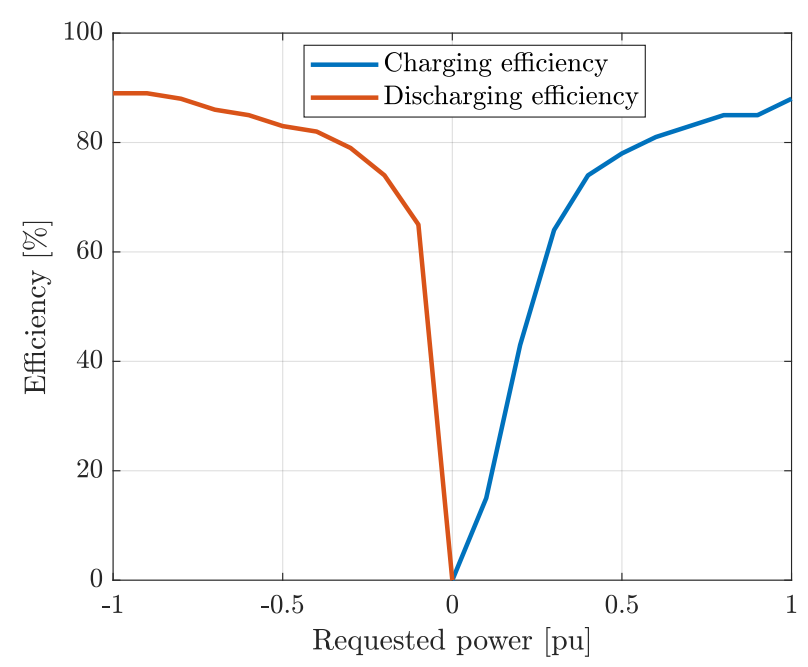

Figure 2: Efficiency characteristics based on real data.

samples are between $49.95 \mathrm{~Hz}$ and $50.05 \mathrm{~Hz}$, which means that a droop curve like the one in Fig. 1 would result in normalized loads below 0.5 for most of the time and consequently low average efficiencies, according to Fig. 2.

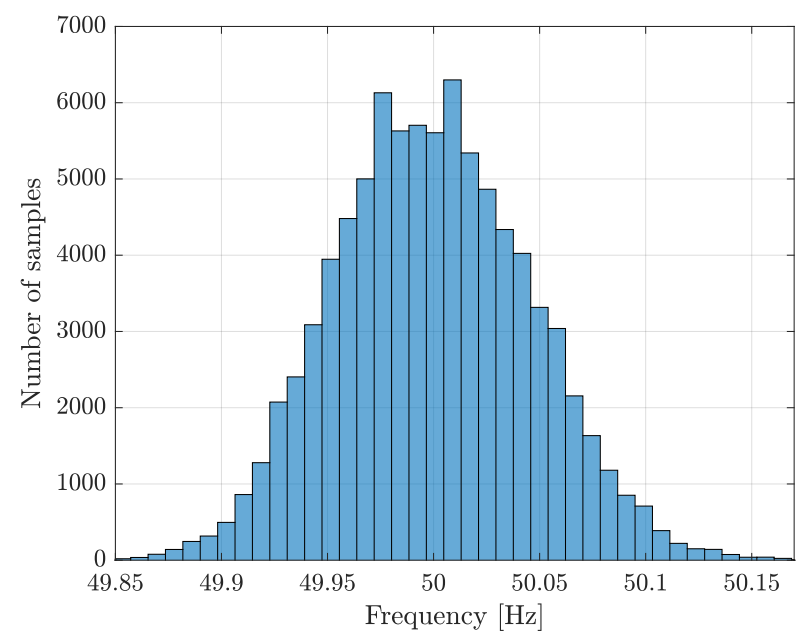

Figure 3: Histogram of frequency values for 10 days.

Other inverters may have significantly higher efficiencies in lower operating points, if they are designed accordingly. Even if in that case a droop-based response with $1 \mathrm{~A}$ steps will not result in very low average efficiency, still the proposed controller can optimize the aggregation's average efficiency under a decentralized control scheme. However, we consider the presented efficiencies as a more realistic case, because they are obtained from actual $\mathrm{V} 2 \mathrm{G}$ chargers performing FNR.

\section{Discretized Decentralized Control}

\section{A. Basic algorithm}

As shown in the following example, a deterministic droop controller with a non-continuous response will always result in a response error, except for the cases where the requested response coincides with a discrete step of the charger's output. 
Consider the case of a 1 A granularity, which corresponds to $0.4 \mathrm{~kW}$ steps for a DC voltage equal to $400 \mathrm{~V}$. If the power response is rounded to its closest value, the response error as a function of the requested power will be as shown in Fig. 4.

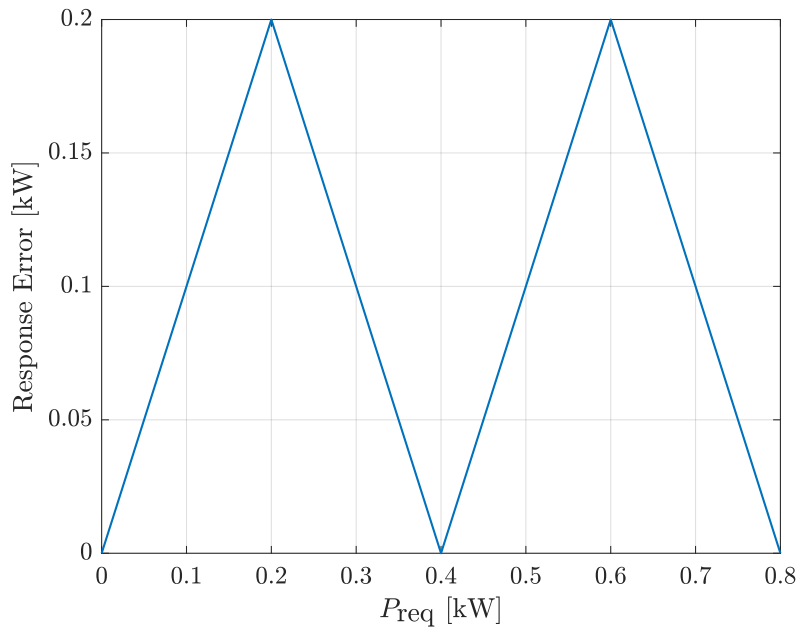

Figure 4: Response error (absolute value) as a function of the requested power.

As already shown, frequency is normally distributed around $50 \mathrm{~Hz}$. If the frequency distribution is discretized in so many steps as in our case, i.e. 25 steps, the resulting probability distributions for each bin can be very well approximated by uniform distributions. Considering a uniform distribution of the frequency within each bin, the distribution of the response errors will retain the triangular shape shown in Fig. 4. It is trivial to show that this results in an average error of $0.1 \mathrm{~kW}$, which for a reserve capacity of $10 \mathrm{~kW}$ is equivalent to a MAPE of $1 \%$. This error is of course independent of the number of EVs and is relatively small; however, it can be drastically reduced by employing a simple stochastic strategy as explained later. The main drawback of this method is that it results in very low efficiencies, since the EVs operate at low loadings most of the time.

We propose a decentralized stochastic controller whose tuning objective is to compromise efficiency and reserve errors, taking into account the size of the EVs aggregation. Stochastic controllers based on random number generations which force loads to operate either at full capacity or to be idle have been proposed in the literature, such as [3], [16]. Our approach differs because it employs an arbitrary discretization of the response to address efficiency and aggregation size. A very fine discretization results in small errors but poor efficiencies. On the other hand, 3 states (idle, fully charging or discharging) will result in high efficiencies but high errors, unless the aggregation is large.

First, the response of each EV is discretized in bins represented by a vector $\mathbf{v}$ in ascending order and normalized per reserve capacity. We define the mapping $\mathrm{g}: \mathbb{R} \rightarrow \mathbb{R}^{2}$, which maps a value $P_{\text {req, } t}$ to bins $i$ and $j$ of the vector $\mathbf{v}$ so that $\mathbf{v}(i) \leq P_{\text {req, } t} \leq \mathbf{v}(j)$. Depending on the calculated $P_{\text {req }, t}$, the controller identifies the 2 bins its response must lie within, calculates a switching probability $p$ and draws a random number. This simple Bernoulli trial, denoted by $h(p)$ and its outcome $b$, will determine the state $s$ of the EV. The control algorithm is illustrated in Algorithm 1.

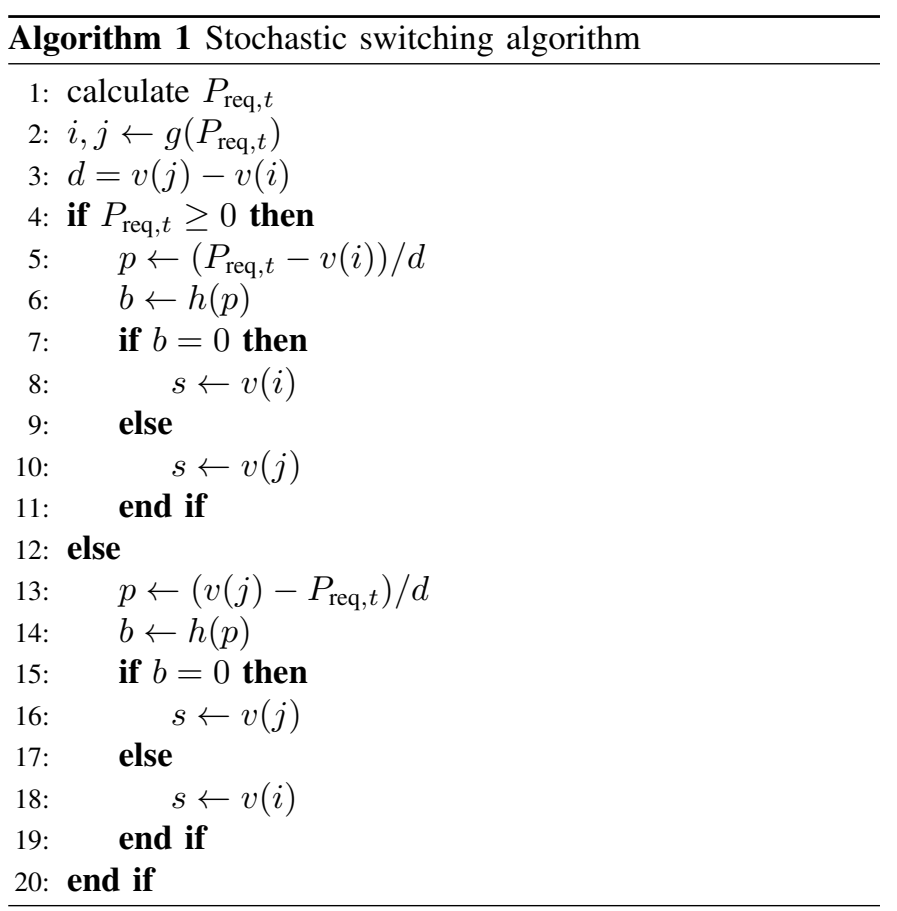

\section{B. Switching minimization}

We presented the basic version of the control algorithm. It is possible to minimize the switching actions of the inverters by modifying the algorithm for the cases where the requested power lies within the same 2 bins in two consecutive time steps. We illustrate the algorithm's modification with an example instead of an algorithm diagram, due to space limitations. Consider the case of two time steps $t_{1}, t_{2}$ where $P_{\text {req }, t_{1}}=0.2$ and $P_{\text {req, } t_{2}}=0.3$ and $\mathbf{v}=[-1,-0.5,0,0.5,1]$. At $t_{1}$, approximately $60 \%$ of the chargers' outputs will be equal to 0 and $40 \%$ equal to $50 \%$. Instead of all the EVs drawing random numbers at $t_{2}$, only a portion of the loads with power equal to 0 have to switch to the next bin; more specifically, these loads will apply the stochastic process with $p=0.1 /(0.5 * 0.6)=33.3 \%$. Similarly, if $P_{\text {req }, t_{2}}=0.1$, then only only a portion of the loads with power equal to $50 \%$ will apply the stochastic process with $p=0.1 /(0.5 * 0.4)=$ $50 \%$. Following similar arguments, the chargers can minimize their switching in the cases of negative $P_{\text {req }, t}$. Note that this algorithm is also decentralized and no coordination is required. Each load will apply this algorithm considering the expected state of the population and not the exact number of EVs in each state, whereas only the change $P_{\text {req, } t_{2}}-P_{\text {req, } t_{1}}$ determines which loads will apply the stochastic process. These modifications in the algorithm can drastically reduce the number of switchings without noticeable increases in the MAPEs, as shown in the following section. 


\section{RESULTS}

We used a real 4 hour frequency sample to assess the performance of the different control strategies. We assume that all EVs are available during reserve provision, as is the case in [12], and provide the maximum reserve capacity, equal to $\pm 10 \mathrm{~kW}$. The chosen frequency sample satisfies two conditions: (a) frequency does not have a significant bias, so that charging and discharging are almost equally represented, and (b) frequency presents a relatively large variance around $50 \mathrm{~Hz}$ so that small frequency deviations are not over-represented. Frequency samples with small variance are expected to yield worse efficiencies when a droop curve is used and our purpose is to make a fair comparison with our proposed controller. The normalized requested power corresponding to the frequency sample is shown in Fig. 5 and the time step used for all simulations is $1 \mathrm{~s}$.

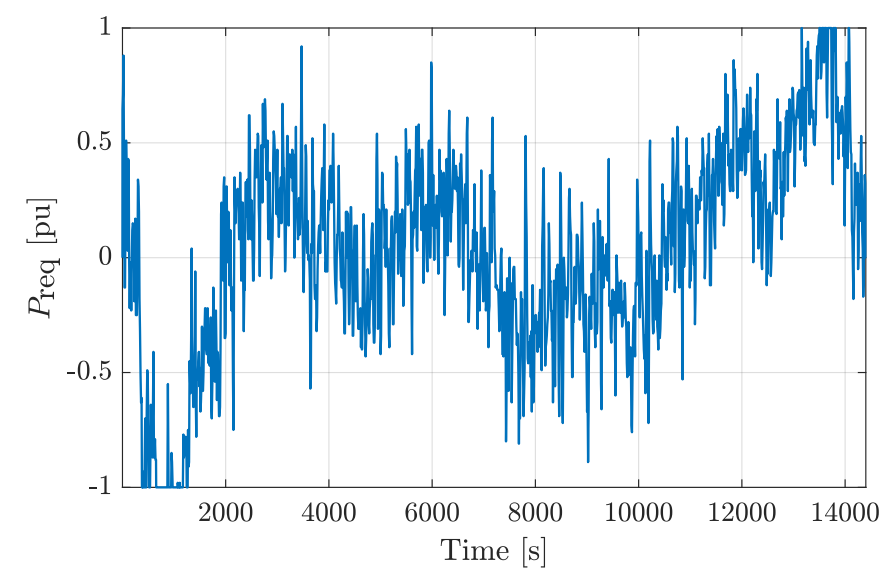

Figure 5: Normalized requested power for a $4 \mathrm{~h}$ frequency sample.

\section{A. Effect of aggregation size and controller granularity on $M A P E$}

We first analyzed the performance of a deterministic controller with a $4 \%$ granularity (corresponding to the $1 \mathrm{~A}$ steps) which simply rounds the requested power to the closest possible power output; we found that it results in a MAPE equal to $1 \%$, as theoretically calculated in Section IV. Due to the deterministic nature of the controller, the error does not depend on the aggregation size.

We then examined the effect of the discretization step on the average reserve error. We used the modified controller which minimizes the switching rate in our simulations. As already explained, a discretization with very small steps is expected to produce very small reserve errors, since any inaccurate number draws have a small impact on the error. On the other hand, large steps are expected to result in larger MAPEs because inaccurate number draws produce relatively large errors. However, as the number of EVs increases, the results of the random-number generations are closer to the expected values and the errors decrease. The reserve MAPEs as a function of the EVs number for 6 different discretization steps are shown in Fig. 6.

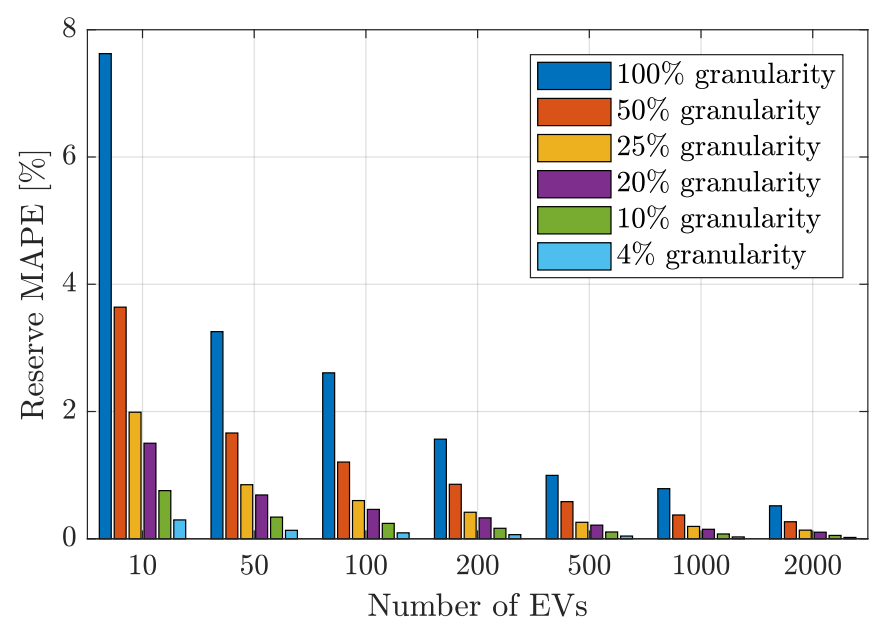

Figure 6: Reserve MAPEs as a function of the EVs number for different granularities of the response.

It is evident that for small aggregations a large granularity results in significant MAPEs. The advantage of using a stochastic controller even for the case of the $4 \%$ granularity is evident by the fact that MAPE decreases from $1 \%$ (deterministic case) to $0.4 \%$ for $10 \mathrm{EVs}$ and $0.17 \%$ for $50 \mathrm{EVs}$. A MAPE of $1 \%$ requires more than $500 \mathrm{EVs}$ for a granularity of $100 \%$ and as few as 50 EVs for a granularity of $25 \%$.

Next, we calculated the MAPEs when the modification for minizing the switchings was not used. A continuous switching is expected to produce smaller MAPEs because at each time step all EVs will draw a random number and respond; when the switching minimization is applied, the EVs switch based on the expected distribution of the EVs between two bins. For smaller aggregation sizes the actual and expected distributions may not be the same (for larger sizes the difference is negligible) and thus the calculated probability may not reflect the ideal probability. However, simulations showed that the exclusion of the modification in the controller results in very small differences in the MAPE and for a size larger than 100 EVs the errors are almost the same. For 10 EVs and $100 \%$ granularity, the modification increases the MAPE from $7.6 \%$ to $7.9 \%$ and for 50 EVs from $3.36 \%$ to $3.4 \%$.

\section{B. Effect of controller granularity on the average efficiency}

To calculate the effect of the controller's granularity on the average efficiency we used the modified algorithm because it significantly reduces the switching actions and it has a minimal effect on the MAPEs. We calculated the average charging and discharging efficiencies for the entire reserve provision duration for each granularity; the results are shown in Fig. 7. We observed that the efficiencies do not depend on the number of the EVs because the stochastic process itself is the same for all loads and on average it doesn't affect efficiency. As already discussed, most frequency samples are distributed close to $50 \mathrm{~Hz}$, which would force the EVs to operate on low loadings if they use a typical droop curve with small steps. This is reflected in the simulation results, where the 
average efficiencies increase significantly as the steps become larger. This can be explained by the fact that when large steps are used, e.g. $50 \%$ or $100 \%$, the EVs will be either idle or charging/discharging at much higher capacities even for small requested powers.

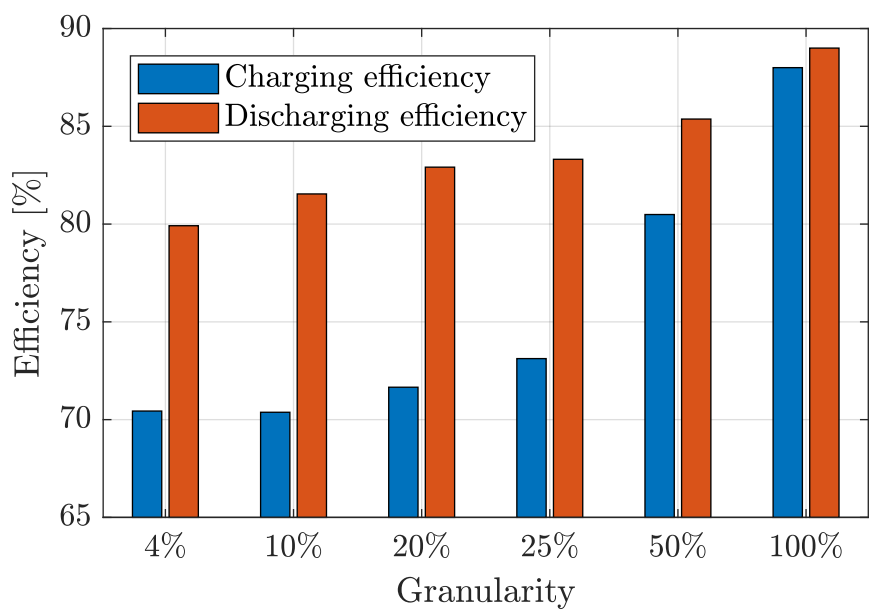

Figure 7: Average charging and discharging efficiencies for the different controller granularities.

To illustrate the effect of such differences on the average EVs SOC when they are offering FNR, we simulated their SOC for 4 different discretizations and for the case without any losses. The average SOC is expressed in pu of $P_{\text {res }}$, i.e. for $P_{\text {res }}=10 \mathrm{~kW}$ a SOC value of 1 corresponds to $10 \mathrm{kWh}$. We show the change of SOC, denoted by $\triangle S O C$, compared to an initial zero value for the different cases in Fig. 8.

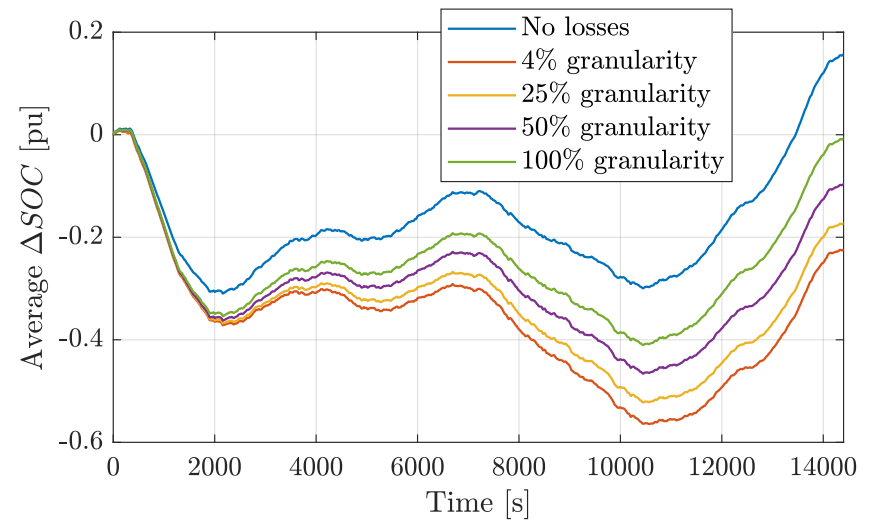

Figure 8: Evolution of the average $\triangle S O C$ for different controller discretizations, compared to a lossless operation.

Notice the effect the different controllers have on the average SOC over a period of 4 hours providing FNR. If no losses occurred, then the average SOC at the end of the period is equal to $0.15 \mathrm{pu}$, or $1.5 \mathrm{kWh}$ for a $P_{\text {res }}=10 \mathrm{~kW}$. Instead of charging with this amount, the EVs would discharge by more than $2 \mathrm{kWh}$ using a droop curve of $1 \mathrm{~A}$ steps, whereas with 3 modes the average SOC would be equal to zero. Notice also the variance in the evolution of the SOC; the larger it is throughout the reserve provision period, the harder it is for the EVs to offer reserves. In other words, the aggregator needs to be more conservative in the amount of offered reserve capacity, so as not to reach the upper or lower battery limits while providing reserves.

\section{Average switching actions}

A potential disadvantage of using a discretized decentralized controller is the frequent switching of the inverters. Usually inverters are designed to handle frequent changes in their output but the impact on the inverters and EV batteries should also be considered when designing the controller. Recognizing the potential wear on the equipment, we proposed a modification of the controller in Section IV to minimize the switching actions. We simulated both control approaches and we present the average switching rates for each granularity in Fig. 9. Note that the average switching rate is presented as a percentage of the time steps, i.e. a rate of $1 \%$ means that an inverter will change state 144 times over 4 hours.

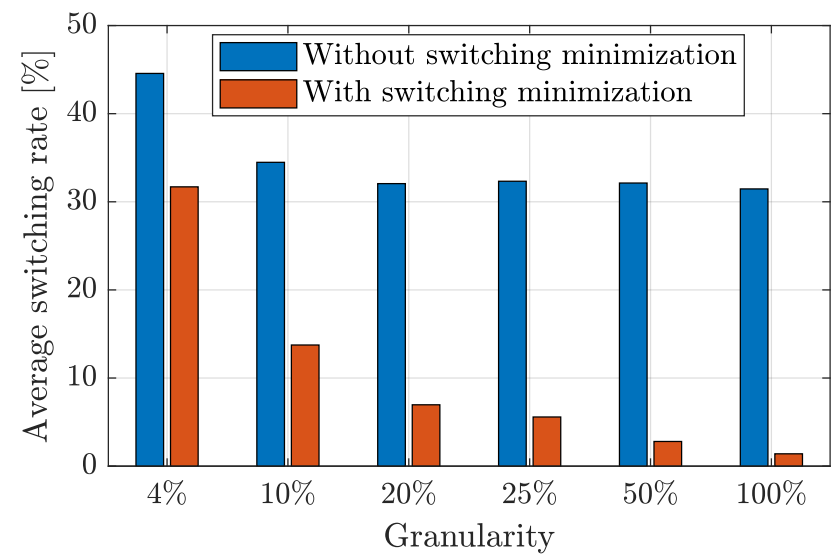

Figure 9: Average switching rates with and without switching minimization.

It is interesting to note that without switching minimization the average switching rate is almost constant and very high (more than 30\%); this means for the control time step of $1 \mathrm{~s}$, then on average an inverter will switch every $3 \mathrm{~s}$, which is a very high rate. If we modify the controller, as explained in Section IV, the switching rates are reduced dramatically, reaching an average value of $1.4 \%$ (or less than 1 switching per minute) if only 3 modes are used. More complicated control approaches may further reduce the switching rates.

\section{Optimizing the controller's discretization steps}

With the proposed decentralized control approach it is possible to define different discretizations, without necessarily having equal distances between two consecutive bins. For example, it is reasonable to design a controller with a finer granularity in higher loadings, which at the same time avoids operating at loadings below 50\%. In this regard, in Fig. 10 the MAPEs and the average efficiencies for 3 different strategies are shown. It is interesting to note the different performance of the controllers for the used discretizations. By taking the $\left[\begin{array}{lllll}-100 & -50 & 0 & 50 & 100\end{array}\right] \%$ discretization as the benchmark, the addition of an intermediate upper state 
equal to $75 \%$ of the capacity has a minimal effect on the average efficiencies but reduces the errors. Additionally, a discretization of $\left[\begin{array}{lllll}-100 & -60 & 0 & 60 & 100\end{array}\right] \%$ of the response results in slightly larger errors compared to the previous case but increases the average efficiencies.
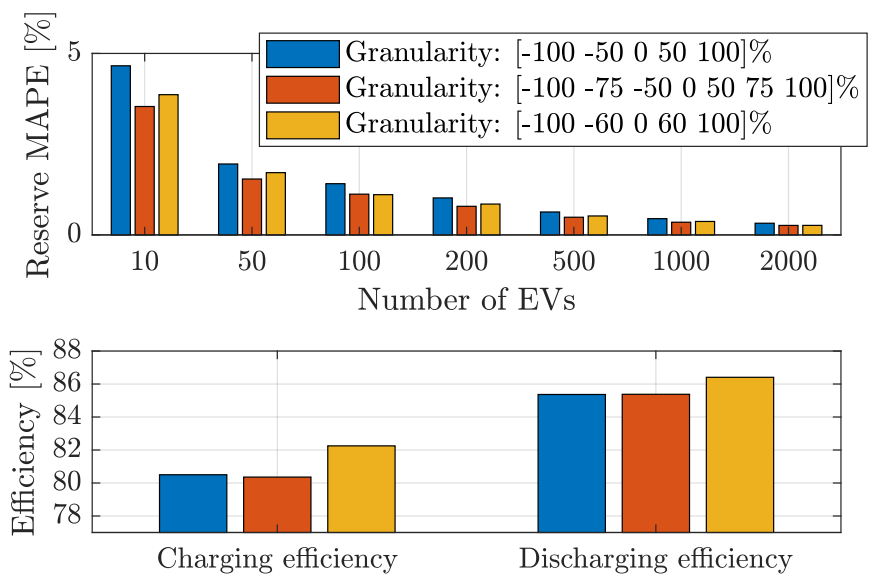

Figure 10: MAPEs and average efficiencies for 3 different discretizations of the controller.

Our purpose is not to present the best discretization for the efficiency values we use in this study, but to show that there are various trade-offs when designing the controllers. In particular, we showed that the efficiency curves, the allowed reserve errors based on the service requirements, the allowed switching rates and the number of EVs must be all taken into account to find the optimal discretization for a given EV fleet offering FNR.

\section{CONCLUSION}

We proposed a stochastic, decentralized controller which relies only on local frequency measurements and whose discretized response can be optimized according to a set of criteria. We showed that a droop-curve response with a 1 A granularity results in low efficiencies and high average switching rates, albeit in low reserve errors. On the other hand, a response with only 3 states results in high efficiencies but unacceptable reserve errors for small EV fleets. The proposed controller, which is also designed to minimize the switching actions of the chargers, can compromise efficiency, average switching rates and reserve errors for a given $\mathrm{EV}$ fleet size.

Thus, if the fleet size does not allow the EVs operator to choose the most efficient response discretization (which is fully charging, idle or fully discharging), it can optimize the discretization based on an efficiency, reserve error and average switching rate trade off. It is interesting to note that since the chargers' efficiency characteristics are highly non-linear, the ideal response discretization which maximizes efficiency and guarantees a maximum reserve error is not trivial to be found and may also depend on the frequency signal characteristics. It is thus necessary to take all the aforementioned factors into account and their effect on performance when optimizing the proposed controller. In our future work we will generalize the controller by considering arbitrary target set-points and nonsymmetrical assigned reserve capacities and we will perform a validation on the proposed controller on real V2G chargers performing FNR under realistic conditions.

\section{ACKNOWLEDGMENTS}

The authors would like to acknowledge the support of the EUDP projects ACES - Across Continents Electric Vehicle Services (grant EUDP17-I-12499, website: www.acesbornholm.eu) and Ecogrid 2.0 (grant 64015-0082, website: www.ecogrid.dk)

\section{REFERENCES}

[1] M. Rezkalla, A. Zecchino, S. Martinenas, A. M. Prostejovsky, and M. Marinelli, "Comparison between synthetic inertia and fast frequency containment control based on single phase evs in a microgrid," Applied Energy, vol. 210, pp. 764 - 775, 2018. [Online]. Available: http://www.sciencedirect.com/science/article/pii/S0306261917308024

[2] K. Knezović, S. Martinenas, P. B. Andersen, A. Zecchino, and M. Marinelli, "Enhancing the role of electric vehicles in the power grid: Field validation of multiple ancillary services," IEEE Transactions on Transportation Electrification, vol. 3, no. 1, pp. 201-209, March 2017.

[3] M. G. Vayá and G. Andersson, "Combined smart-charging and frequency regulation for fleets of plug-in electric vehicles," in 2013 IEEE Power Energy Society General Meeting, July 2013, pp. 1-5.

[4] E. Sortomme and K. W. Cheung, "Intelligent dispatch of electric vehicles performing vehicle-to-grid regulation," in 2012 IEEE International Electric Vehicle Conference, March 2012, pp. 1-6.

[5] S. Izadkhast, P. Garcia-Gonzalez, P. Frias, and P. Bauer, "Design of Plugin Electric Vehicle's Frequency-Droop Controller for Primary Frequency Control and Performance Assessment," IEEE Transactions on Power Systems, vol. PP, no. 99, pp. 1-1, 2017.

[6] A. Molina-Garcia, F. Bouffard, and D. S. Kirschen, "Decentralized demand-side contribution to primary frequency control," IEEE Transactions on Power Systems, vol. 26, no. 1, pp. 411-419, Feb 2011.

[7] M. R. V. Moghadam, R. Zhang, and R. T. B. Ma, "Distributed frequency control via randomized response of electric vehicles in power grid," IEEE Transactions on Sustainable Energy, vol. 7, no. 1, pp. 312-324, Jan 2016.

[8] S. Izadkhast, P. Garcia-Gonzalez, and P. Frías, "An aggregate model of plug-in electric vehicles for primary frequency control," IEEE Transactions on Power Systems, vol. 30, no. 3, pp. 1475-1482, May 2015.

[9] G. Wenzel, M. Negrete-Pincetic, D. E. Olivares, J. MacDonald, and D. S. Callaway, "Real-time charging strategies for an electric vehicle aggregator to provide ancillary services," IEEE Transactions on Smart Grid, vol. PP, no. 99, pp. 1-1, 2017.

[10] H. Liu, Z. Hu, Y. Song, and J. Lin, "Decentralized vehicle-to-grid control for primary frequency regulation considering charging demands," IEEE Transactions on Power Systems, vol. 28, no. 3, pp. 3480-3489, 2013.

[11] "Road vehicles — vehicle to grid communication interface — part 1: General information and use-case definition," https://www.iso.org/obp/ ui/\#iso:std:iso:15118:-1:ed-1:v2:en, 2013.

[12] ENEL, "Nissan, enel and nuvve operate world's first fully commercial vehicle-to-grid hub in denmark," https://www.enel.com, 2016.

[13] Energinet.dk, "Ancillary services to be delivered in denmark tender conditions." https://en.energinet.dk/-/media/Energinet/El-RGD/ Dokumenter/Ancillary-services-to-be-delivered-in-Denmark.pdf, 2017.

[14] A. Thingvad, C. Ziras, J. Hu, and M. Marinelli, "Assessing the energy content of system frequency and electric vehicle charging efficiency for ancillary service provision," 2017 Proceedings of the 52nd International Universities Power Engineering Conference, 2017.

[15] http://www.statnett.no.

[16] E. Vrettos, C. Ziras, and G. Andersson, "Fast and Reliable Primary Frequency Reserves From Refrigerators with Decentralized Stochastic Control," IEEE Transactions on Power Systems, vol. 32, no. 4, pp. 2924 2941, 2017. 